Role of proteins in controlling selenium nanoparticle size

This article has been downloaded from IOPscience. Please scroll down to see the full text article.

2011 Nanotechnology 22195605

(http://iopscience.iop.org/0957-4484/22/19/195605)

View the table of contents for this issue, or go to the journal homepage for more

Download details:

IP Address: 128.178.41.120

The article was downloaded on 25/03/2011 at 09:39

Please note that terms and conditions apply. 


\title{
Role of proteins in controlling selenium nanoparticle size
}

\author{
J Dobias, E I Suvorova and R Bernier-Latmani ${ }^{1}$ \\ Environmental Microbiology Laboratory, EPFL, Station 6, 1015 Lausanne, Switzerland \\ E-mail: rizlan.bernier-latmani@epfl.ch
}

Received 27 August 2010, in final form 27 December 2010

Published 23 March 2011

Online at stacks.iop.org/Nano/22/195605

\begin{abstract}
This work investigates the potential for harnessing the association of bacterial proteins to biogenic selenium nanoparticles (SeNPs) to control the size distribution and the morphology of the resultant SeNPs. We conducted a proteomic study and compared proteins associated with biogenic SeNPs produced by E. coli to chemically synthesized SeNPs as well as magnetite nanoparticles. We identified four proteins (AdhP, Idh, OmpC, AceA) that bound specifically to SeNPs and observed a narrower size distribution as well as more spherical morphology when the particles were synthesized chemically in the presence of proteins. A more detailed study of AdhP (alcohol dehydrogenase propanol-preferring) confirmed the strong affinity of this protein for the SeNP surface and revealed that this protein controlled the size distribution of the SeNPs and yielded a narrow size distribution with a three-fold decrease in the median size. These results support the assertion that protein may become an important tool in the industrial-scale synthesis of SeNPs of uniform size and properties.
\end{abstract}

S] Online supplementary data available from stacks.iop.org/Nano/22/195605/mmedia

\section{Introduction}

Biological systems can produce a tremendous variety of potential nanomaterial products. If fully deciphered, these biological systems could be harnessed for industrial nanomaterial manufacturing. Biologically aided synthesis could help decrease the consumption of energy and toxic chemicals, opening the path for more environmentally friendly green manufacturing (Pearce et al 2008).

Bacteria, among all biological systems, are well known to produce metal and metal oxide nanoparticles (NPs) of various compositions, sizes and morphologies. For instance, Bacillus selenitireducens can reduce tellurium as tellurate or tellurite to rosette-aggregated $\mathrm{Te}(0)$ rods of $30 \mathrm{~nm} \times$ $200 \mathrm{~nm}$ and selenium as selenite or selenate to $\operatorname{Se}(0) 200 \mathrm{~nm}$ spherical particles (Oremland et al 2004, Baesman et al 2007); Shewanella oneidensis MR-1 reduces tellurium to 50-80 nm spherical particles (Klonowska et al 2005); Magnetospirillum magneticum AMB-1 produces 30-120 nm cubic magnetic particles (Lang and Schuler 2006) and Veillonella atypica produces $30 \mathrm{~nm} \mathrm{ZnSe}$ and CdSe particles (Pearce et al 2008).

However, there is a significant knowledge gap in our collective understanding of the mechanism of formation of

\footnotetext{
1 Author to whom any correspondence should be addressed.
}

those NPs: it is unclear how the control of the final product is achieved. This knowledge gap precludes mass production on an industrial scale using bacterially based nanomanufacturing. Therefore, there is a salient need to develop a mechanistic understanding of the processes leading to the formation of metallic nanoparticles by bacteria.

Bacterial synthesis of metallic NPs is often achieved by a reduction step followed by a precipitation step with the latest composed of two parts: nucleation and crystal growth. To date, only the reduction step has been studied extensively and the biological processes responsible for nucleation and crystal growth are not fully understood. Several studies provide evidence that proteins might play a key role in the nucleation and crystal growth of bacteriogenic metal NPs. A bacterial protein-cytochrome $c_{3}$-was found to reduce selenate $\left(\mathrm{SeO}_{4}^{2-}\right)$ in aqueous solution leading to the formation of one-dimensional chain-like aggregates of monoclinic selenium nanoparticles (SeNPs) (Abdelouas et al 2000). Secondly, in magnetosomes of the magnetotactic bacterium, Magnetospirillum magneticum AMB-1, membrane proteins are tightly bound to the magnetic NPs (Gorby et al 1988, Leinfelder et al 1988, Tanaka et al 2006) and single proteins (Mms6 and BSA) were shown to be able to control the shape of the final particles (Arakaki et al 2003, Kaur et al 
2009). Similarly, the rate of crystal growth and the morphology of Au NPs was shown to be controlled by proteins. These proteins, which are able to constrain the speed of Au NP crystal growth as well as to direct particle morphology, were identified from a random phage-display peptide library (Brown 1992, Brown et al 2000). Finally, short peptide-based biopanning techniques (Sano and Shiba 2003, Lower et al 2008) showed the strong adhesion of some peptides to titanium NP surfaces.

In order to better understand the role of proteins in controlling the formation of nanoparticles, we studied the reduction of selenite to elemental selenium by $E$. coli. This microorganism offers the advantage of being well-studied and genetically tractable, which allows the ready use of genetic engineering and molecular biology. Additionally, it is able to reduce tetravalent and hexavalent selenium to elemental selenium, $\operatorname{Se}(0)$.

Selenium ( $\mathrm{Se}$ ) is an element of interest for electronics and photonics applications. Its attractiveness stems from its high refractive index (>2.5) (Jeong and Xia 2005) and its high reactivity: the reduction and disproportionation of elemental selenium allow the coating of selenium nanostructures with other metals $(\mathrm{Pt}, \mathrm{Cd})$ and can be used to produce core/shell nanostructures as inverted opaline lattices (Jeong and Xia 2005) or other functional materials such as silver selenide (Jeong and Xia 2005). In order to be used at an industrial scale, it requires an efficient and affordable method of production of monodispersed nanospheres of amorphous selenium (a-Se).

To date, several synthetic techniques exist to produce spherical SeNPs. These include: (a) exposing selenious acid to gamma-radiation (Zhu et al 1996), (b) reducing selenious acid by various reagents such as hydrazine $\left(\mathrm{N}_{2} \mathrm{H}_{4}\right)$ (Dimitrijevic and Kamat 1988), (c) oxidizing selenide ions electrochemically (Franklin et al 1990), (d) crystallizing meltquenched amorphous selenium (Zhang et al 1995), (e) using a reverse micelle method (Johnson et al 1999) or (f) using laser ablation (Jiang et al 2003). However, these techniques have limitations. The most significant of which are that they do not yield the narrow size distributions (size variation of less than 5\%) (Jeong and Xia 2005) that are important for industrial applications and that they produce particles that are subject to extreme photocorrosion (Dimitrijevic and Kamat 1988). In order to overcome the limitations of the previous techniques (high temperature, high pressure or use of catalysts), biologically based, semi-synthetic methods have been explored to produce nanomaterials (Abdelouas et al 2000).

This work focuses on pinpointing the role of naturally occurring E. coli proteins in controlling the size and size distribution of SeNPs. We identified proteins that bind strongly to biogenic SeNPs. The focus on strongly binding proteins was based on the presumption that binding is required for the protein to impact the SeNPs. We selected a single protein for a detailed study of its effect on the morphology and size distribution of these NPs.

The long-term goal of this work is to identify proteins that play a role in the bacteria-dependent biomineralization of selenium and other metals, to unravel their binding mechanism and to help develop protocols for industrial applications. A substantial advantage of protein-based approaches as opposed to whole-cell approaches is that there is no need to maintain live cultures for the process of NP synthesis. Furthermore, we believe that the biologically based, semi-synthetic production of NPs may be a viable economic alternative to existing nanomaterial production processes due to the added value of avoiding the production and use of environmentally hazardous chemicals and promoting green manufacturing.

\section{Materials and methods}

All chemicals were of analytical grade and obtained from Sigma-Aldrich (Basel, Switzerland), unless otherwise stated.

\subsection{Bacterial strains and growth conditions}

In this study, we used Escherichia coli K-12 obtained from DSMZ (DSM-No. 498). Bacterial cultures were grown aerobically at $30^{\circ} \mathrm{C}$ in liquid Luria-Bertani (LB) broth (10 $\mathrm{g} \mathrm{l}^{-1}$ Tryptone, $10 \mathrm{~g} \mathrm{l}^{-1}$ sodium chloride, $5 \mathrm{~g} \mathrm{l}^{-1}$ yeast extract) in Erlenmeyer flasks $(250 \mathrm{ml})$ containing $125 \mathrm{ml}$ medium, inoculated from a $10 \%(\mathrm{v} / \mathrm{v})$ overnight culture in LB and placed on a rotary shaker (140 rpm).

\subsection{Production of zero-valent SeNPs}

To test the hypothesis that proteins are associated to NPs in vivo and that they can bind them in vitro, we produced biogenic SeNPs (BioSeNPs) using E. coli K-12 and chemogenic SeNPs (ChSeNPs) according to a protocol modified from Lin and Wang (2005). The method as it was published (Lin and Wang 2005) presented two major limitations: the occasional presence of sulfur polymer structures and a strong dependency of NP size on incubation time. To circumvent these issues, we used a concentration of selenious acid ( $\mathrm{Se}(\mathrm{IV})$ ) varying from 0.7 to $5.2 \mathrm{mM}$, a ratio of sodium thiosulfate $\left(\mathrm{Na}_{2} \mathrm{O}_{3} \mathrm{~S}_{2}\right)$ to selenious acid varying from $1: 30$ to $1: 150$ and fixed the reaction time to $18 \mathrm{~h}$ in a $0.01 \mathrm{M}$ (final concentration) sodium dodecyl sulfate (SDS) solution. Purified protein or E. coli cell-free extract were added at a final concentration of $0.1 \mathrm{mg} \mathrm{ml}^{-1}$ in appropriate experiments. The speed of the reaction and the size of the particles are controlled by the ratio of Se(IV) to sodium thiosulfate. Particle size can be visually estimated based on the colour of the solution due to the size-specific plasmon phenomenon (Lin and Wang 2005).

BioSeNPs were produced as follows: an overnight culture of $E$. coli $\mathrm{K}-12$ was supplemented with filter-sterilized selenious acid $\left(\mathrm{H}_{2} \mathrm{SeO}_{3}\right)$ as the source of $\mathrm{Se}(\mathrm{IV})$ to a final concentration of $4 \mathrm{mM}$ and incubated for two days. Se(IV) reduction to $\mathrm{Se}(0)$ was visible with the appearance of a dark red colouration in the culture. We measured the reduction of $\mathrm{Se}(\mathrm{IV})$ by sampling the culture over time, filtering the samples with a $0.2 \mu \mathrm{m}$ pore diameter syringe filter followed by filtration with a $0.02 \mu \mathrm{m}$ pore diameter syringe filter. The filtrate $(1 \mathrm{ml})$ was acidified with $0.1 \mathrm{~N} \mathrm{HNO}_{3}(9 \mathrm{ml})$ and measured for total Se in solution by inductively coupled plasma optical emission spectroscopy (ICP-OES; Perkin Elmer Optima 3000).

To separate BioSeNPs from biomass, cells were lysed by adding $\mathrm{NaOH}$ to a final concentration of $1 \mathrm{~N}$ and heating the 
suspension in a boiling water bath for $20 \mathrm{~min}$. The resultant mixture was amended with n-hexane and placed in a separatory funnel. The solvent phase contained the biomass and the aqueous phase contained the NPs. The $\mathrm{pH}$ of the collected aqueous fraction containing the SeNPs was then lowered to 7.2 using $6 \mathrm{M} \mathrm{HCl}$ and NPs were collected by centrifugation using an Eppendorf $\circledast 5415 \mathrm{R}$ centrifuge (16000 relative centrifugal force (rcf), room temperature (RT), $30 \mathrm{~min}$ ), washed three times with Milli-Q water (18 M $\Omega \mathrm{cm}$ water) and stored in Milli-Q water for further use. BioSeNPs free of biomass are hereafter abbreviated BioSeNPsBF.

\subsection{Cell-free extract (CFX) of E. coli $K-12$}

E. coli cells were grown until the mid logarithmic phase (OD600 $=0.4-0.6)$, transferred to $50 \mathrm{ml}$ centrifuge tubes, centrifuged with a Beckman Coulter Avanti J-26XP centrifuge (3000 rcf, $15 \mathrm{~min}, 4^{\circ} \mathrm{C}$ ) and washed twice with phosphate buffered saline (PBS). The cell pellet was frozen at $-80^{\circ} \mathrm{C}$ if not used immediately. Cells were resuspended in ice-cold $100 \mathrm{mM}$ Tris-Cl pH $7.4(10 \mathrm{ml} / 40 \mathrm{ml}$ of cell culture $)$ and kept on ice. They were sonicated (Branson sonifier 150D, Branson ultrasonic corporation, CT, USA) on ice at $100 \mathrm{~W}$ five times for $5 \mathrm{~min}$ ( $4 \mathrm{~s}$ pulse, $2 \mathrm{~s}$ pause) and the temperature was monitored to remain under $20^{\circ} \mathrm{C}$. After each cycle of $5 \mathrm{~min}$, cells were cooled down to $4^{\circ} \mathrm{C}$. Cells were observed under an optical microscope to verify the efficiency of sonication. Unbroken cells and cell debris were removed by centrifugation (16000 rcf, $30 \mathrm{~min}, 4^{\circ} \mathrm{C}$ ). The supernatant was aliquoted into $1 \mathrm{ml}$ samples and stored at $-80^{\circ} \mathrm{C}$. Protein concentration was measured using a Bradford assay from Bio-Rad (Munich, Germany) according to the manufacturer's protocol.

\subsection{Protein-NPs association}

In order to identify the proteins that are natively associated with SeNPs, cells of $E$. coli K-12 that had reduced Se(IV) to $\mathrm{Se}(0)$ were ultrasonicated to release the SeNPs and the lysate was centrifuged in an $80 \%$ sucrose solution. The heavy fraction containing the BioSeNP was separated from the light ones and washed with $100 \mathrm{mM}$ Tris $\mathrm{pH} 7.4$ to remove sucrose.

We also tested the association of proteins present in E. coli $\mathrm{K}-12$ cell-free extract with ChSeNP, BioSeNPsBF and magnetite (Fe(II)/Fe(III) oxide) nanoparticles (FeNPs). FeNPs are available commercially as a nanopowder made of spherical particles $(<50 \mathrm{~nm})$. FeNPs were washed and resuspended in Milli-Q water. We mixed cell-free extract with ChSeNP, BioSeNPBF or FeNPs to a final ratio of $1-1.6 \mathrm{mg} \mathrm{ml}^{-1}$ of proteins to $0.7-1.0 \mathrm{mg}$ of NPs and agitated overnight on a rotary shaker. SeNPs were collected by centrifugation (16000 rcf, $4{ }^{\circ} \mathrm{C}, 30 \mathrm{~min}$ ) and FeNPs were collected using a magnet.

To resolve the protein composition, samples were mixed with gel loading buffer containing (final concentration): $50 \mathrm{mM}$ Tris-HCl (pH 6.8), $100 \mathrm{mM}$ 1,4-dithiothreitol (DTT), $2 \%$ SDS, $10 \%$ glycerol and $0.01 \%$ bromophenol blue. Samples were heated at $95^{\circ} \mathrm{C}$ for $5 \mathrm{~min}$ to denature the proteins and subjected to SDS-polyacrylamide gel electrophoresis (SDSPAGE) in a $12 \%$ (wt/vol) polyacrylamide gel. The gel was stained with 'ProtoBlue ${ }^{\mathrm{TM}}$ Safe' from National Diagnostics (Atlanta, USA).

\subsection{Stripping-off proteins from NPs}

When proteins were found associated with NPs, the strength of the association was tested by a series of increasingly denaturing treatments. NPs were mixed with E. coli cellfree extract at a ratio of $1 \mathrm{mg}$ to $1-1.5 \mathrm{mg} \mathrm{ml}^{-1}$, respectively, and left overnight at room temperature on a rotator. NPs were collected by centrifugation $\left(16000 \mathrm{rcf}, 4{ }^{\circ} \mathrm{C}, 30 \mathrm{~min}\right)$ and the supernatant transferred to a fresh tube. The pelleted NPs were washed twice with $100 \mathrm{mM}$ Tris-Cl pH 7.4 to remove free proteins. Subsequently NPs were treated either sequentially or individually with six different solutions (from least to most denaturing): (1) $2 \%$ Triton X-100, (2) $2 \%$ SDS, (3) a solution composed of $7 \mathrm{M}$ urea, $2 \mathrm{M}$ thiourea, $4 \%$ CHAPS (Biochemica, Applichem GmbH, Germany), $40 \mathrm{mM}$ Tris base (abbreviated 'Urea 7 M'), (4) 10\% SDS, (5) 10\% SDS and boiling for $10 \mathrm{~min}$ and (6) 10\% SDS and boiling for $30 \mathrm{~min}$. For the individually treated samples, the NPs were resuspended in the adequately stringent solution and gently shaken for $20 \mathrm{~min}$ at room temperature on a rotary shaker. For the sequentially treated samples, each step was performed as follows: an aliquot was collected and washed with the previous solution. The remaining sample was centrifuged (16000 rcf, $10 \mathrm{~min}, \mathrm{RT}$ ) to collect the NPs and the supernatant was stored. The NPs were resuspended in the washing solution of the next stringency and agitated (20 min, RT) on a rotary shaker. The collected fractions (aliquot, supernatants and NPs) were characterized by the Bradford assay and SDS-polyacrylamide gel electrophoresis (SDS-PAGE) techniques (Laemmli 1970).

\subsection{Protein identification}

Proteins within a given sample were separated by SDS-PAGE. To identify individual proteins, the bands of interest were cut out, sliced into $1 \mathrm{~mm}$ slices and sent for protein identification to EPFL's protein core facility (www.pcf.epfl.ch). Samples were reduced and alkylated with dithioerythritol (DTE) and iodoacetamide (IAA), respectively, in order to reduce and block disulfide bonds. Samples were dried and in-gel digested with Trypsin for at least $12 \mathrm{~h}$ at $37^{\circ} \mathrm{C}$. Peptides were then extracted from gel pieces and concentrated by speed-vac evaporation. Samples were finally resuspended and analysed by liquid chromatography ion trap mass spectrometry (LCIT-MS/MS). Reverse phase LC separation was performed on a nano-HPLC quaternary pump (Rheos 2200) at a flow rate of $700 \mathrm{nl} \mathrm{min}{ }^{-1}$ using a C18 capillary column $(100 \mu \mathrm{m}$ id $\times$ $100 \mathrm{~mm}$ ). MS analysis was performed on a Finnigan/Thermo LTQ Ion-Trap MS instrument. An E. coli UniProt (SwissProt) sub-database and the Matrix Science Ltd. Mascot search engine were used to perform identifications using the mass fragments detected. Mascot's discriminating factors $p$ and ionic score (IS) were chosen such that $p<10^{-6}$ and IS $>40$.

\subsection{Electron microscopy (EM)}

Samples for the electron microscopy study were prepared by washing NPs and depositing a drop of the sample suspension on a carbon-coated copper grid. Samples were air-dried at room temperature overnight in a dust-free box. 
Local analysis to investigate the morphology and structure of particles was performed by transmission electron microscopy (TEM), electron diffraction and x-ray energy dispersive spectroscopy (EDS) in a FEI CM 300FEG-UT analytical transmission electron microscope $(300 \mathrm{kV}$ field emission gun). The images were recorded with a Gatan 797 slow scan CCD camera (1024 pixels $\times 1024$ pixels $\times$ 14 bits) and processed with the Gatan Digital Micrograph 3.11.0 software. The chemical composition of particles was obtained from $x$-ray EDS in scanning transmission electron microscopy (STEM) mode with 2-50 nm diameter electron probes and interpreted with the INCA (Oxford) software.

Scanning electron microscopy (SEM) observations were done with a FEI XL30 FEG microscope. The secondary electron SEM images were obtained at 2, 5 or $25 \mathrm{kV}$ accelerating voltage depending on the size of the particles.

\subsection{Particle size measurement}

We used EM to measure sizes of individual particles but, in addition, in order to have representative values of particle populations, we used two dynamic light scattering (DLS) instruments: (1) a Beckman Coulter LS 13320 laser diffraction particle size analyser that can measure spherical particles from $40 \mathrm{~nm}$ to $2 \mathrm{~mm}$ and (2) a Malvern Zetasizer nano ZS, which has a size range of $0.3 \mathrm{~nm}-10 \mu \mathrm{m}$. When shown, the number of particles as a percentage of the total measured particles was computed using a refractive index of 2.6 and an absorption coefficient of 0.5 (Dowd 1951).

\subsection{Ultraviolet-visible (UV-vis) characterization}

To compare the plasmon phenomenon of the NPs, we measured the absorbance of NPs in the UV-vis region with a Shimadzu UV-vis recording spectrophotometer UV-2501PC.

\subsection{AdhP cloning}

Alcohol dehydrogenase propanol-preferring (AdhP) is one of the proteins identified in the cell-free extract to bind strongly to SeNPs. We tested its effect on ChSeNPs during NP formation. To do so, we produced purified protein by cloning and overexpressing it in E. coli. We cloned AdhP with an Invitrogen ${ }^{\mathrm{TM}}$ (Basel, Switzerland) Champion ${ }^{\mathrm{TM}}$ pETD200/TOPO ${ }^{\circledR}$ expression kit by strictly following the supplied protocol. The gene encoding AdhP was generated by polymerase chain reaction (PCR) amplification using genomic DNA from E. coli $\mathrm{K}-12$ as the template with the primers DJadhp_F2 (5' -cac cAT GAA GGC TGC AGT TGT TA-3') and DJ-adhp_R2 (5'-TTA GTG ACG GAA ATC AAT CAC CAT GC-3') and New England Biolabs (Ipswich, MA, USA) Vent polymerase.

Positive colonies containing the cloned gene were selected on kanamycin $\left(50 \mu \mathrm{g} \mathrm{ml}^{-1}\right)$ agar plates, the plasmid was purified using a Sigma-Aldrich (Basel, Switzerland) GeneElute $^{\mathrm{TM}}$ plasmid mini prep kit and sequenced by Fasteris SA (Geneva, Switzerland).

Bacteria overexpressing AdhP (BL21AdhP) were grown in Invitrogen ${ }^{\mathrm{TM}}$ MagicMedia supplemented with kanamycin $\left(50 \mu \mathrm{g} \mathrm{ml}^{-1}\right)$. Cells were collected, washed and lysed according to a protocol from the Bio-Rad (Reinach, Switzerland) Profinia protein purification system that was used to purify the His-tagged AdhP (His-ADHP). Invitrogen ${ }^{\mathrm{TM}}$ InVision $^{\mathrm{TM}}$ Histag in-gel stain was used to specifically stain His-ADHP on protein gels. Finally the N-terminal 6xHIS fragment was removed with Invitrogen ${ }^{\mathrm{TM}}$ EnterokinaseMax ${ }^{\mathrm{TM}}$ system by strictly following the supplied protocol (we used 1 unit of $\mathrm{EKMax}^{\mathrm{TM}}$ for the His-tag cleavage) and the purified protein is referred to as pAdhP hereafter.

\subsection{AdhP activity assay}

To test the proper conformation and the activity of pAdhP, we used the alcohol dehydrogenase enzymatic assay protocol from Sigma-Aldrich (Sigma-Aldrich 1998) based on Kägi and Vallee (1960). It consists of following the reduction of $\beta$ NAD to NADH by measuring the absorbance of the latter at $340 \mathrm{~nm}$ overtime. One unit (U) is equal to the production of $1 \mu \mathrm{mol} \mathrm{min} \mathrm{m}^{-1}$ of NADH and the specific activity of the enzyme

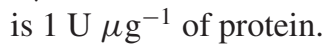

\section{Results and discussion}

\subsection{Characterization of bacteriogenic NPs}

ICP-OES measurements (figure S1 available at stacks.iop.org/ Nano/22/195605/mmedia) showed that E. coli could reduce $4 \mathrm{mM}$ of selenite over two days. In the absence of cells or in the presence of heat-killed cells, the reduction does not occur. No toxic effect of selenite or SeNPs was observed as spent medium supplemented with yeast extract and peptone did not impair the growth of a fresh E. coli inoculum, and the inoculation of fresh medium with bacteria grown in the presence of selenite for more than two days showed bacterial growth (data not shown). The appearance of a dark red colour indicated the formation of amorphous elemental selenium particles (figures S2 and S3 available at stacks.iop.org/Nano/22/195605/mmedia). Electron microscopy analysis revealed spheroidal particles (figure S2-A available at stacks.iop.org/Nano/22/195605/mmedia) with no crystalline structure (figure S2-B available at stacks.iop.org/ Nano/22/195605/mmedia) and a size range of 10-90 nm. DLS measurements gave an average size of $62 \pm 15 \mathrm{~nm}$ (figure S4 available at stacks.iop.org/Nano/22/195605/mmedia), which is an overestimate as the Beckmann DLS instrument used has a detection limit of $40 \mathrm{~nm}$ at the lower end.

\subsection{Protein identification}

The goal was to identify proteins potentially involved in the biomineralization of $\mathrm{Se}(0)$. Our approach was to assay for the association of proteins to biogenic SeNPs. The assumption inherent in this approach is that proteins involved in nanoparticle formation are tightly associated with the produced NPs (Abdelouas et al 2000, Arakaki et al 2003, Aryal and Benson 2007, Brown 1992, Sano and Shiba 2003, Lower et al 2008). The assay involved growing $E$. coli bacteria in the presence of selenite until the appearance of a brick red colour representative of the presence of $\operatorname{Se}(0)$ particles. The $\operatorname{Se}(0)$ NPs 


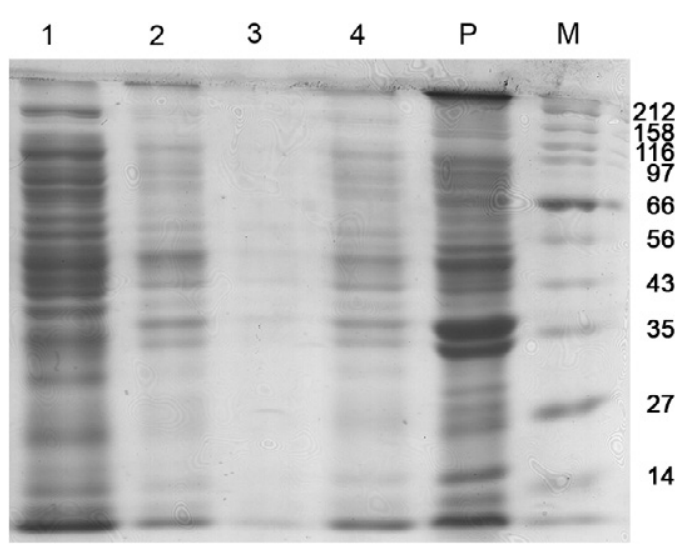

Figure 1. 12\% SDS-PAGE of fractions 1-4 of the sucrose separation (figure S5 available at stacks.iop.org/Nano/22/195605/mmedia), P (pellet fraction from lysed cells) and $\mathrm{M}$ (protein ladder with sizes in kDa). Fractions 2 and 4 include SeNPs.

and associated proteins were then collected by lysing the cells via ultrasonication and centrifuging the lysate through an $80 \%$ sucrose solution leading to four fractions (figure S5 available at stacks.iop.org/Nano/22/195605/mmedia). Fractions 1 and 3 were transparent and considered NP-free as opposed to fractions 2 and 4 (figure S5 available at stacks.iop.org/Nano/ 22/195605/mmedia), which were orange-red and therefore considered to contain a significant amount of SeNPs. Fraction 4 was a brick red SeNP pellet which was the focus of further work. The protein content in these fractions was analysed by SDS-PAGE (figure 1). The proteins of fraction 1 were distributed throughout the entire size range and were representative of the entire proteome. This distribution pattern differed significantly from those of fractions 2 and 4 (figure 1) and fraction 3 did not exhibit any proteins. Fractions 2 and 4 differed from fractions 1,3 and $P$, suggesting a specific enrichment of certain proteins through their association with SeNPs.

To test the strength of protein binding to NPs, the NPs from fraction 4 were washed with increasingly stringent denaturing solutions. Some proteins remained attached to the NPs even after 10 min of boiling in $10 \%$ SDS (figure 2) which implies a very strong interaction between these proteins and the NPs. The experiment was repeated with (a) BioSeNPsBF exposed to CFX (figure S6 available at stacks.iop.org/Nano/ 22/195605/mmedia), (b) FeNPs exposed to CFX (figure S7 available at stacks.iop.org/Nano/22/195605/mmedia) and (c) ChSeNPs formed in the presence of CFX (figure S8 available at stacks.iop.org/Nano/22/195605/mmedia). The FeNPs were used to differentiate between specific and non-specific association of proteins to NPs. The bands delimited by black boxes in figures 2 and S6-S8 (available at stacks.iop.org/Nano/22/195605/mmedia) were cut out of the gel and proteins were identified by nano-LC-IT-MS/MS. Results of protein identification are given in tables S1-S4 (available at stacks.iop.org/Nano/22/195605/mmedia).

None of the identified NP associated proteins are known to be involved in selenium or iron metabolism. Instead, they are related to energy production and carbohydrate or fatty acid

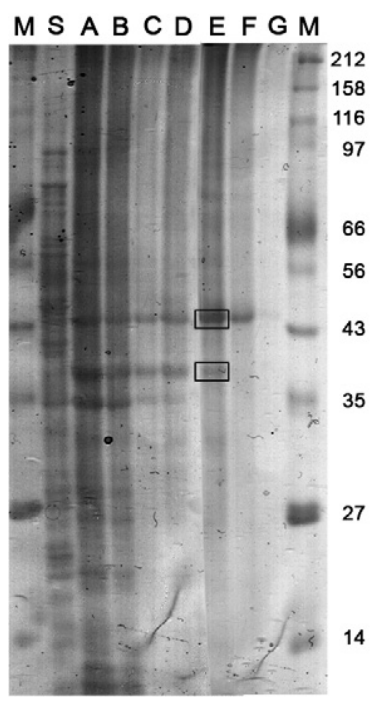

Figure 2. 12\% SDS-PAGE of BioNPs from E. coli grown in selenite: A (no treatment), B (Triton 2\%), C (SDS 2\%), D (urea 7M), E (SDS 10\%), F (boiled 10 min in SDS 10\%), G (boiled 30 min in SDS $10 \%), S$ (supernatant from centrifuged lysed cells) and $\mathrm{M}$ (protein ladder with sizes in $\mathrm{kDa}$ ). The square boxes are the bands that were cut out and identified by mass spectrometry.

metabolism. ADHP, ACEA, ENO, KPYK1, IDH and GLPK require metallic cofactors (respectively $\mathrm{Zn}$, divalent cations, $\mathrm{Mg}, \mathrm{Mg}-\mathrm{K}, \mathrm{Mn}-\mathrm{Mg}$ and $\mathrm{Zn}$ ) and DCEA, ASTC and TNAA require non-metallic cofactors (pyridoxal phosphate). One could speculate that the binding to cofactors could explain their strong association with SeNPs.

Only two proteins were found to be common to all tested conditions (including FeNPs): elongation factor Tu (EFTU) and 3-oxoacyl synthase (FABB), suggesting a non-specific binding of those proteins to metallic and metal oxide NPs.

Four proteins were found to be associated specifically and solely to SeNPs (table 1). These four proteins vary in size (36$48 \mathrm{kDa}$ ), in function (enzyme or structural protein) as well as in isoelectric point (4.58-5.94). Additionally, there is no obvious similarity in amino acid sequence between the four proteins. Thus, no evidence of a clear mechanism leading to the binding of these specific proteins to SeNPs can be gleaned from the information currently available.

\subsection{Role of CFX proteins in SeNPs formation}

In section 3.2 we showed that some proteins are strongly attached to SeNPs. In order to identify the potential effect of proteins on SeNPs, we chemically synthesized SeNPs (ChSeNPs at a 1:30 ratio of Se(IV) to sodium thiosulfate) in the presence and the absence of E. coli CFX and performed TEM, selected area electron diffraction (SAED) and EDS analyses. The CFX appears to restrict the size distribution of NPs yielding a more tightly controlled size distribution of $106.7 \pm$ 8.7 nm (figure $3 \mathrm{~B}$ ) versus 10-90 nm (figure $3 \mathrm{~A}$ ). Furthermore, NPs formed in the presence of CFX are almost perfectly spherical as opposed to the ones formed in its absence. In both cases, NPs are made of non-crystalline selenium (data not shown). Unfortunately, the extreme effect of CFX on ChSeNP 


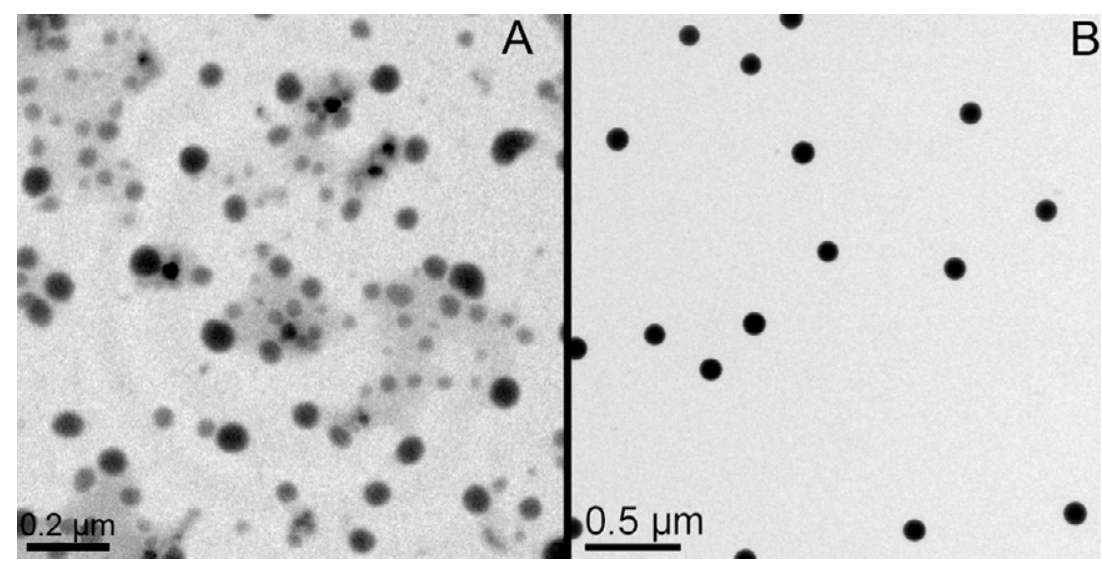

Figure 3. TEM of chemogenic NPs produced at a 1:30 Se(IV):thiosulfate ratio in the absence (A) and presence (B) of cell-free extract.

Table 1. Identified proteins specific to SeNPs.

\begin{tabular}{|c|c|c|c|c|c|}
\hline Name & & Size $[\mathrm{kDa}]$ & $\mathrm{IP}^{\mathrm{a}}$ & Cofactor & Function \\
\hline ACEA & Isocitrate lyase & 48 & 5.16 & divalent cations & $\begin{array}{l}\text { Glyoxylate and } \\
\text { dicarboxylate } \\
\text { metabolism }\end{array}$ \\
\hline IDH & $\begin{array}{l}\text { Isocitrate } \\
\text { dehydrogenase } \\
\text { [NADP] }\end{array}$ & 46 & 5.15 & $\mathrm{Mg}$ or $\mathrm{Mn}$ & $\begin{array}{l}\text { Tricarboxylic acid cycle } \\
\text { and } \\
\text { glyoxylate bypass }\end{array}$ \\
\hline OMPC & $\begin{array}{l}\text { Outer membrane } \\
\text { protein C } \\
\text { precursor (Porin } \\
\text { ompC) }\end{array}$ & 40 & 4.58 & & Passive pore formation \\
\hline ADHP & $\begin{array}{l}\text { Alcohol } \\
\text { dehydrogenase, } \\
\text { propanol-preferring }\end{array}$ & 36 & 5.94 & $\mathrm{Zn}$ & $\begin{array}{l}\text { Fermentation } \\
\text { (Aldehyde/ketone } \\
\text { formation) }\end{array}$ \\
\hline
\end{tabular}

${ }^{\mathrm{a}}$ Isoelectric point

synthesis was difficult to reproduce for a more detailed study. One of the main issues was the composition of CFX. Because there is variation in the exact composition of the CFX as a function of the batch of grown bacteria, it is not practically feasible to use CFX as an experimental reagent. Nonetheless, every batch of CFX tested decreased the size distribution range of synthesized SeNPs but to varying extents.

\subsection{AdhP effect on SeNPs}

In order to tackle the mechanism of binding of proteins to NPs in a more tractable experimental system, we resolved to study proteins individually. We tested the effect of a single purified protein on the reduction-nucleation-growth process during chemical production of elemental selenium. We chose to work with AdhP for two reasons: (1) it was found to be associated only to SeNPs (table 1) and (2) we can ensure that its three-dimensional conformation is correct by quantifying its enzymatic activity. We tested the binding ability of His-AdhP to BioSeNPs, the enzymatic activity of pAdhP and the effect of pAdhP on the formation of ChSeNPs synthesized at a selenite to sodium thiosulfate ratio of 1-150.

As stated previously, we selected AdhP as the target protein to study due to its preferential binding to SeNPs as determined from incubations with $E$. coli $\mathrm{CFX}$. We confirmed this characteristic of AdhP by quantifying the binding of the recombinant protein His-AdhP to BioSeNPs by protein gel (figure 4). As is evident from the protein gel, lanes corresponding to purified His-AdhP and BioSeNPs exposed to His-AdhP both show a clear band at the correct size. In contrast, the supernatant derived from the centrifugation of a suspension of BioSeNPs and His-AdhP shows no evidence for the protein, suggesting the removal of His-AdhP from solution through binding to SeNPs. Thus, there is overwhelming evidence for the strong binding of His-AdhP to BioSeNPs.

For a meaningful comparison of the effect of recombinant AdhP and native E. coli AdhP on NP formation, the two proteins have to be structurally similar. To test the similarity of the proteins, we used the enzymatic activity as an indicator of their spatial conformation. Therefore, we measured the enzymatic activity of pAdhP and found 108 and $126 \mathrm{U}^{*}$ min $-1 * \mathrm{mg}^{-1}$ for ethanol and propanol substrates, respectively. These values are in the range of reported activities for alcohol dehydrogenase enzymes from various species [unit: $\mathrm{U}^{*} \mathrm{~min}^{-1}$ $*^{*} \mathrm{mg}^{-1}$ ]: 43 for E. coli, 40-184 for Drosophila melanogaster and 210-7300 for Saccharomyces cerevisiae (Shafqat et al 1999, Blandino et al 1997, Bozcuk et al 2004). Therefore, we concluded that the recombinant protein was active and that its spatial conformation corresponded to that of the native protein. Hence, we could reliably compare in vivo and in vitro systems. 


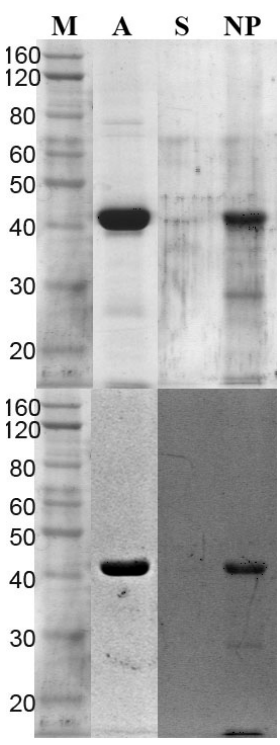

Figure 4. Binding of His-AdhP to bacteriogenic SeNPs: (A) Purified His-AdhP, (S) supernatant, (NP) NP exposed to His-AdhP and (M) protein ladder (sizes are in $\mathrm{kDa}$ ). The upper gel was stained with coomassie blue whereas the lower gel was stained with InVision ${ }^{\mathrm{TM}}$ His-tag stain.

We evaluated the effect of pAdhP on ChSeNPs formation. We synthesized ChSeNPs in the presence of pAdhP and compared those to ChSeNPs synthesized in the absence of protein. We observed a three-fold decrease of the average NP size in the presence of pAdhP (figure 5 and S9). Specifically, SeNPs produced in the presence of protein were $122 \pm 24 \mathrm{~nm}$ in

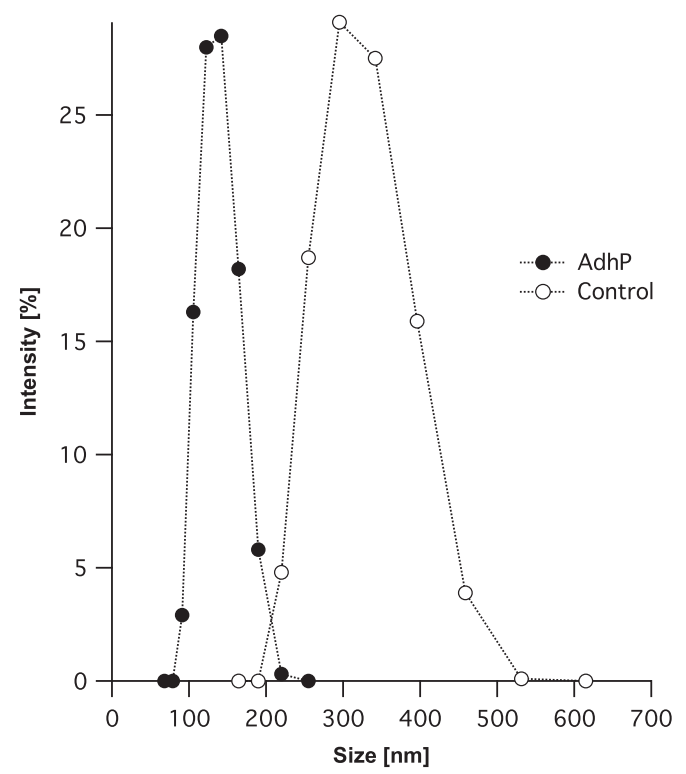

Figure 5. Zetasizer DLS size measurement of ChSeNP synthesized in the presence (AdhP) and in the absence (control) of pAdhP.

size whereas those produced in its absence were $319 \pm 57 \mathrm{~nm}$ in size. The size distribution measured by DLS is consistent with the absorption spectrum of the SeNPs produced by the two treatments (figure S12 available at stacks.iop.org/Nano/ 22/195605/mmedia): the untreated SeNPs are larger and show a prominent peak at $\sim 620 \mathrm{~nm}$ whereas the NPs synthesized in the presence of pAdhP are smaller with no clear peak but a broad shoulder at $\sim 480 \mathrm{~nm}$. In both cases the particles

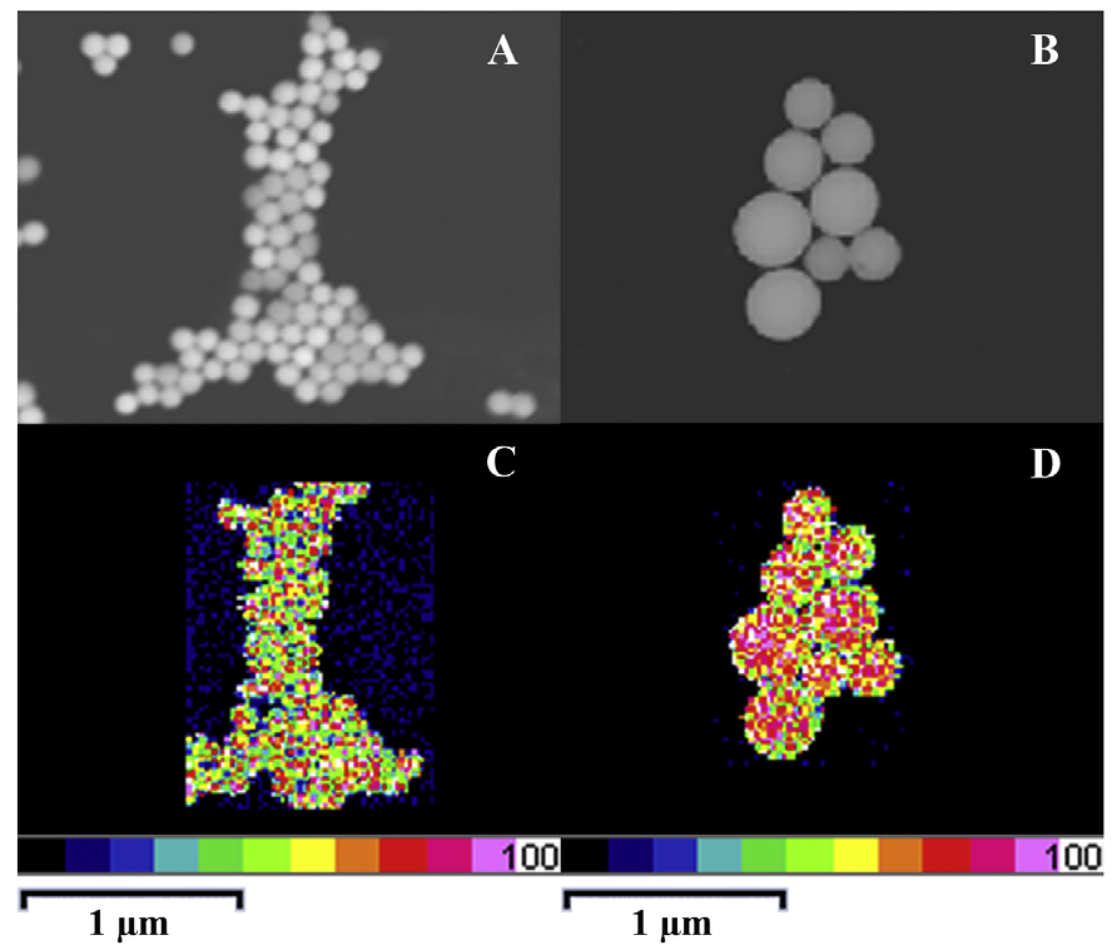

Figure 6. STEM ((A), (B)) and EDS selenium mapping ((C), (D)); colours represent the abundance percentage of the mapped element from $0 \%$ (black) to $100 \%$ (white)) of ChSeNP produced in the presence of AdhP ((A), (C)) or in the absence of AdhP ((B), (D)). 
were spherical as is shown in SEM micrographs (figure S10 and $\mathrm{S} 11$ available at stacks.iop.org/Nano/22/195605/mmedia). EDS mapping confirmed that the SeNPs were made only of selenium (figure 6) and SAED showed no crystalline structure for the NPs (data not shown). Overall, these data show that among possible effects of proteins on NPs (e.g. shape, size, crystallinity), only the size distribution was modified-albeit three-fold-when pAdhP was present during the chemical reduction of selenite by sodium thiosulfate. This result also suggests that the decrease in size distribution range of SeNPs obtained with CFX (figure 3) may be the combined effect of several proteins rather than the sole impact of pAdhP.

Other researchers have previously suggested that proteins can have an impact on the shape of SeNPs (Kaur et al 2009). Combined with the results described here, those findings point to a complex and important role for proteins in biological and chemical $\mathrm{Se}(0)$ nanoparticle formation. Taken together, our results and previously published ones (Abdelouas et al 2000, Brown 1992, 1997, Kaur et al 2009) strongly suggest that proteins may play a major role in controlling the characteristics of NPs from biological or chemical origin. While the ability to control size and shape using proteins may provide a basis for exploring the use of biomolecules in the synthesis of SeNPs at larger than laboratory scale, it also warrants continued and targeted research into the mechanism of binding of these protein to the surface of NPs.

\section{Conclusions}

In biological systems, the synthesis of NPs by bacteria is equivalent to simple reactions occurring in a complex chemical environment. These environments are rich in biomolecules such as proteins, polysaccharides, nucleic acids, fatty acid or sugars. In this work, we studied the interaction between proteins and metallic NPs and the role of proteins in the formation of NPs. In preliminary experiments, we observed that CFX (a complex matrix of biomolecules) was able to affect the size distribution of ChSeNPs by narrowing their size distribution. We also observed that in biological matrices, SeNPs and FeNPs are associated with a large number of proteins and that several of these are strongly bound to the NPs. The identification of these strongly associated proteins revealed that, among the identified proteins, none has a reported function that is related to NP formation or metal reduction. These proteins are primarily implicated in energy, carbohydrate or fatty acid metabolism but do not share chemical properties such as isoelectric point, cofactor or size. We conclude that the binding ability of the proteins depends either on their spatial configuration or/and on physicochemical properties of their amino acid. We tested the effect of a single purified protein, AdhP, on the formation of ChSeNPs and found a significant effect on size distribution: a three-fold decrease in the average size of ChSeNPs.

Overall, this work shows that the control of the size distribution of synthetic SeNPs produced in a simple aqueous system and under standard ambient temperature and pressure conditions is possible through harnessing the interactions of naturally occurring proteins with these NPs. The proteinderived control of NP size could have great implications for industrial-scale production.

\section{Acknowledgments}

We would like to acknowledge the Protein Core Facility (PCF), the Protein Elution Core Facility (PECF) and the Centre Interdisciplinaire de Microscopie Électronique (CIME) at EPFL for equipment use and technical advice.

\section{References}

Abdelouas A, Gong W L, Lutze W, Shelnutt J A, Franco R and Moura I 2000 Using cytochrome $\mathrm{c} 3$ to make selenium nanowires Chem. Mater. 12 1510-2

Arakaki A, Webb J and Matsunaga T 2003 A novel protein tightly bound to bacterial magnetic particles in magnetospirillum magneticum strain AMB-1 J. Biol. Chem. 278 8745-50

Aryal B P and Benson D E 2007 Polyhistidine fusion proteins can nucleate the growth of CdSe nanoparticles Bioconjug. Chem. 18 585-9

Baesman S M, Bullen T D, Dewald J, Zhang D, Curran S, Islam F S, Beveridge T J and Oremland R S 2007 Formation of tellurium nanocrystals during anaerobic growth of bacteria that use $\mathrm{Te}$ oxyanions as respiratory electron acceptors Appl. Environ. Microbiol. 73 2135-43

Blandino A, Caro I and Cantero D 1997 Comparative study of alcohol dehydrogenase activity in flor yeast extracts Biotechnol. Lett. 19 651-4

Bozcuk A N, Sumer S, Ozsoy E D and Arisoy M 2004 Age-related enzyme activity in different genotypes of alcohol dehydrogenase (ADH) in Drosophila melanogaster Biogerontology 5 243-7

Brown S 1992 Engineered iron oxide-adhesion mutants of the Escherichia coli phage lambda receptor Proc. Natl Acad. Sci. USA 89 8651-5

Brown S 1997 Metal-recognition by repeating polypeptides Nat. Biotechnol. 15 269-72

Brown S, Sarikaya M and Johnson E 2000 A genetic analysis of crystal growth J. Mol. Biol. 299 725-35

Dimitrijevic N M and Kamat P V 1988 Photoelectrochemistry in particulate systems. 8. Photochemistry of colloidal selenium Langmuir 4 782-4

Dowd J J 1951 Optical properties of selenium Proc. Phys. Soc. B 64 783-9

Franklin T C, Adeniyi W K and Nnodimele R 1990 The Electro-oxidation of some insoluble inorganic sulfides, selenides, and tellurides in cationic surfactant-aqueous sodium hydroxide systems J. Electrochem. Soc. 137 480-4

Gorby Y A, Beveridge T J and Blakemore R P 1988 Characterization of the bacterial magnetosome membrane J. Bacteriol. 170 $834-41$

Jeong U and Xia Y 2005 Synthesis and crystallization of monodisperse spherical colloids of amorphous selenium $A d v$. Mater. 17 102-6

Jiang Z Y, Xie Z X, Xie S Y, Zhang X H, Huang R B and Zheng L S 2003 High purity trigonal selenium nanorods growth via laser ablation under controlled temperature Chem. Phys. Lett. 368 425-9

Johnson J A, Saboungi M L, Thiyagarajan P, Csencsits R and Meisel D 1999 Selenium nanoparticles: a small-angle neutron scattering study J. Phys. Chem. B 103 59-63

Kaur G, Iqbal M and Bakshi M S 2009 Biomineralization of fine selenium crystalline rods and amorphous spheres J. Phys. Chem. C 113 13670-6

Klonowska A, Heulin T and Vermeglio A 2005 Selenite and tellurite reduction by Shewanella oneidensis Appl. Environ. Microbiol. 71 5607-9 
Laemmli U K 1970 Cleavage of structural proteins during the assembly of the head of bacteriophage T4 Nature 227 680-5

Lang C and Schuler D 2006 Biogenic nanoparticles: production, characterization, and application of bacterial magnetosomes J. Phys.: Condens. Matter 18 S2815-28

Leinfelder W, Forchhammer K, Zinoni F, Sawers G, Mandrand-Berthelot M A and Böck A 1988 Escherichia coli genes whose products are involved in selenium metabolism J. Bacteriol. 170 540-6

Lin Z-H and Wang C R 2005 Evidence on the size-dependent absorption spectral evolution of selenium nanoparticles Mater. Chem. Phys. 92 591-4

Lower B H, Lins R D, Oestreicher Z, Straatsma T P,

Hochella M F Jr, Shi L and Lower S K 2008 In vitro evolution of a peptide with a hematite binding motif that may constitute a natural metal-oxide binding archetype Environ. Sci. Technol. 42 3821-7

Oremland R S, Herbel M J, Blum J S, Langley S, Beveridge T J, Ajayan P M, Sutto T, Ellis A V and Curran S 2004 Structural and spectral features of selenium nanospheres produced by Se-respiring bacteria Appl. Environ. Microbiol. 70 52-60

Pearce C I, Coker V S, Charnock J M, Pattrick R A D, Mosselmans J F W, Law N, Beveridge T J and Lloyd J R 2008 Microbial manufacture of chalcogenide-based nanoparticles via the reduction of selenite using Veillonella atypica: an in situ EXAFS study Nanotechnology 19155603

Sano K and Shiba K 2003 A hexapeptide motif that electrostatically binds to the surface of titanium J. Am. Chem. Soc. 125 14234-5

Shafqat J, Hoog J O, Hjelmqvist L, Oppermann U C T, Ibanez C and Jornvall H 1999 An ethanol-inducible MDR ethanol dehydrogenase/acetaldehyde reductase in Escherichia coli-structural and enzymatic relationships to the eukaryotic protein forms Eur. J. Biochem. 263 305-11

Sigma-Aldrich 1998 Alcohol dehydrogenase assay www. sigmaaldrich.com/etc/medialib/docs/Sigma/ General_Information/alcohol_dehydrogenase.Par.0001.File.tmp/ alcohol_dehydrogenase.pdf

Tanaka M, Okamura Y, Arakaki A, Tanaka T, Takeyama H and Matsunaga T 2006 Origin of magnetosome membrane: proteomic analysis of magnetosome membrane and comparison with cytoplasmic membrane Proteomics 6 5234-47

Zhang H Y, Hu Z Q and Lu K 1995 Transformation from the amorphous to the nanocrystalline state in pure selenium Nanostruct. Mater. 5 41-52

Zhu Y J, Qian Y T, Hai H A and Zhang M W 1996 Preparation of nanometer-size selenium powders of uniform particle size by gamma-irradiation Mater. Lett. 28 119-22 